\title{
Posterior Circulation Intervention but Mind the Spinal Cord!
}

\author{
Shahyan Mohsin Siddiqui1,® \\ Karthik Kulanthaivelu1,๑ \\ 1Department of Neuroimaging and Interventional Radiology, \\ National Institute of Mental Health and Neurosciences, Bengaluru, \\ Karnataka, India \\ 2Department of Neurosurgery, National Institute of Mental Health \\ and Neurosciences, Bengaluru, Karnataka, India
}

Hima Pendharkar ${ }^{1}$ Indira Devi Bhagavatula²

\begin{abstract}
Address for correspondence Chandrajit Prasad, DM, Department of Neuroimaging and Interventional Radiology, National Institute of Mental Health and Neurosciences, Bengaluru, Karnataka 560029, India (e-mail: chandrajitt2@gmail.com).
\end{abstract}

J Clin Interv Radiol ISVIR 2021;5:186-189.

\begin{abstract}
Keywords

- posterior circulation endovascular intervention

- spinal cord infarction

Spinal cord infarction $(\mathrm{SCl})$, a rare complication following endovascular treatment of posterior circulation aneurysms, is infrequently recognized in the periprocedural period. We illustrate the same with digital subtraction angiography and diffusion-weighted magnetic resonance imaging in this series of two cases. Categorically, the anterior spinal artery was patent, post-procedure, in both instances. Likely, guiding catheter-induced local flow alterations initiates the pathogenesis. A subsequent insufficiency of radiculomedullary artery (RMA) reinforcements is the probable mechanism leading to hemodynamic compromise. Our observations further reiterate that periprocedural antiplatelet therapy is conceivably ineffective in preventing $\mathrm{SCl}$. Besides, advanced age and uncontrolled hypertension could potentially exacerbate the periprocedural RMA compromise. Due vigilance is required to recognize this rare and potentially life-threatening complication in posterior circulation neurovascular intervention.
\end{abstract}

\section{Introduction}

Spinal cord infarction (SCI) complicating endovascular embolization of posterior circulation aneurysms is rare and is infrequently recognized in periprocedural setting. ${ }^{1}$ This report of two cases highlights the clinical and imaging findings in two adults who underwent endovascular therapy for posterior circulation aneurysms with their procedural outcome complicated by SCI.

\section{Case Report}

\section{Patient 1}

A 54-year-old male presented with a holocranial headache of 2 days duration. Imaging with a noncontrast-enhanced computed tomography (CT) brain and CT angiogram revealed a
modified-Fisher Grade 4 subarachnoid hemorrhage (SAH) and right superior cerebellar artery (SCA) aneurysm. Digital subtraction angiography (DSA) confirmed a superiorly directed SCA aneurysm (dome-4.9 x $3.6 \mathrm{~mm}$, neck-2.5 mm). Left vertebral artery (VA) angiogram revealed anterior spinal artery (ASA) and multiple radiculomedullary arteries (RMA) arising from V4 and V2 segments, respectively. Systemic heparinization was initiated with $5000 \mathrm{IU}$ of unfractionated heparin $(80 \mathrm{IU} / \mathrm{kg})$ as a bolus and 1,000 IU additionally every following hour. Through right femoral access, the left VA was cannulated with a $6 \mathrm{~F}$ long guiding sheath $(0.88$ inch internal diameter (ID), $5 \mathrm{~F}$ vertebral catheter, 0.035 inch coaxial system with the $6 \mathrm{~F}$ guiding sheath eventually parked at the ostium of V1 segment and maintained with a heparinized saline flush. The $5 \mathrm{~F}$ catheter was replaced with a $6 \mathrm{~F}$ distal access catheter with 0.72 ID that was navigated over a 0.035 -inch hydrophilic
DOI https://doi.org/ $10.1055 / \mathrm{s}-0041-1728988$ ISSN 2457-0214
(C) 2021. Indian Society of Vascular and Interventional Radiology. This is an open access article published by Thieme under the terms of the Creative Commons Attribution-NonDerivative-NonCommercial-License, permitting copying and reproduction so long as the original work is given appropriate credit. Contents may not be used for commercial purposes, or adapted, remixed, transformed or built upon. (https://creativecommons.org/licenses/by-nc-nd/4.0/).

Thieme Medical and Scientific Publishers Pvt. Ltd. A-12, 2nd Floor, Sector 2, Noida-201301 UP, India 
guidewire into the V2 segment of the left VA. Following slow intra-arterial infusion of $2 \mathrm{mg}$ of nimodipine over 10 minutes, it was connected to a continuous nimodipine flush for the rest of the procedure. No stasis of contrast was noted post-connection of nimodipine flush. The aneurysm was cannulated with 0.0165 -inch ID microcatheter, and coil embolization of the aneurysm was successfully performed. Post-extubation, the patient was unable to maintain oxygen saturation and was reintubated. On examination, both upper limbs had reduced power; on immediate magnetic resonance imaging (MRI) evaluation, restricted diffusion was evident in ASA territory involving the $\mathrm{C} 2$ to $\mathrm{C} 7$ level of the cervical cord ( - Fig. 1). A review of the post-embolization angiogram reaffirmed the patency of the ASA. Over the next 48 hours, the weakness progressed to involve his lower limbs. Later, he developed difficulty in breathing due to diaphragmatic paralysis and required continued mechanical ventilation and hence an extended admission in the intensive care unit. He eventually succumbed to a respiratory infection after 3 months.

\section{Patient 2}

A 56-year-old female, a chronic hypertensive, presented with modified Fisher Grade 3SAH following rupture of a posteriorly and superiorly directed basilar top aneurysm (neck-3.9 mm; dome-8.2 X $5.2 \mathrm{~mm}$ ) with multiple blebs. Left VA angiogram revealed ASA arising from the V4 segment. An iatrogenic dissection of the left petrous internal carotid artery (ICA) occurred during the diagnostic angiography. Systemic heparinization, vasodilator administration, and long access were achieved as with the previous case with the long-guiding sheath and guiding catheters in similar positions eventually. No stasis of contrast was noted post-connection of nimodipine flush. Balloon-assisted coil embolization of the basilar top aneurysm was successfully completed. Stenting (LEO STENT (Balt, Montmorency, France) 5.5 x $55 \mathrm{~mm}$ ) of the petrous ICA dissection was performed following occlusion of the aneurysm. Following extubation, the patient was unable to maintain saturation and had to be reintubated. Decreased spontaneous movement of the left upper and lower limb was observed. In view of a ruptured aneurysm, the patient was not preloaded with antiplatelets, and tirofiban infusion was initiated. As per protocol, she was planned for bridge dual antiplatelet therapy. Approximately 2 hours later, there was deterioration in her motor status progressing to left hemiplegia. MRI brain evaluation did not reveal a diffusion abnormality or evidence of new hemorrhage. Chest radiography, done later that day, showed a collapse consolidation of the basal segments of the left lung with elevated left hemidiaphragm-suggesting diaphragmatic paralysis. MRI of the spinal cord was performed; it showed pencil-shaped T2 hyperintensity along the left hemicord involving C2 to C4 segments in the ASA territory. Diffusion-weighted imaging depicted restricted diffusion in the same region. Besides, a small focus of restricted diffusion was also present in the right hemicord (C4 level) in posterior spinal artery territory (-Fig. 2). A review of the post-procedural DSA revealed patent ASA as well as the opacification of the RMA reinforcements. Left diaphragmatic paralysis was confirmed on fluoroscopy,

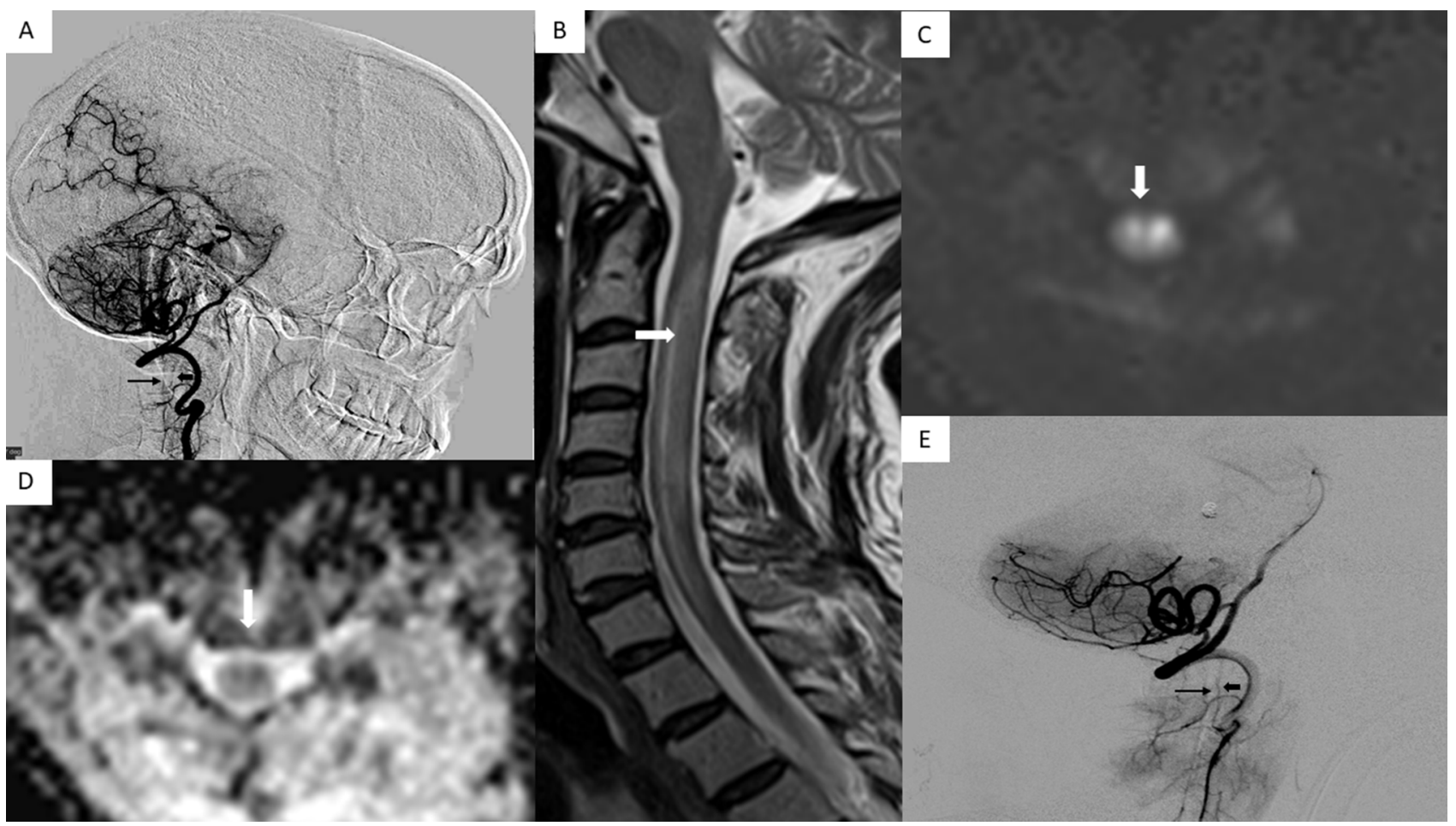

Fig. 1 (A) Left vertebral artery, lateral view demonstrating anterior spinal artery (ASA) (thin black arrow)and right superior cerebellar artery aneurysm (curved black arrow), radiculomedullary artery (RMA) reinforcement (thick black arrow). (B) T2-weighted sagittal image of spinal cord demonstrating long segment (C2-C7) hyperintensity with a pencil-like configuration (white arrow). (C and D) Trace diffusion-weighted imaging and apparent diffusion coefficient map respectively depicting the left hemicord infarction (white arrow). (E) Post-embolization acquisition reveals patent ASA (thin black arrow), RMA reinforcement (thick black arrow). 


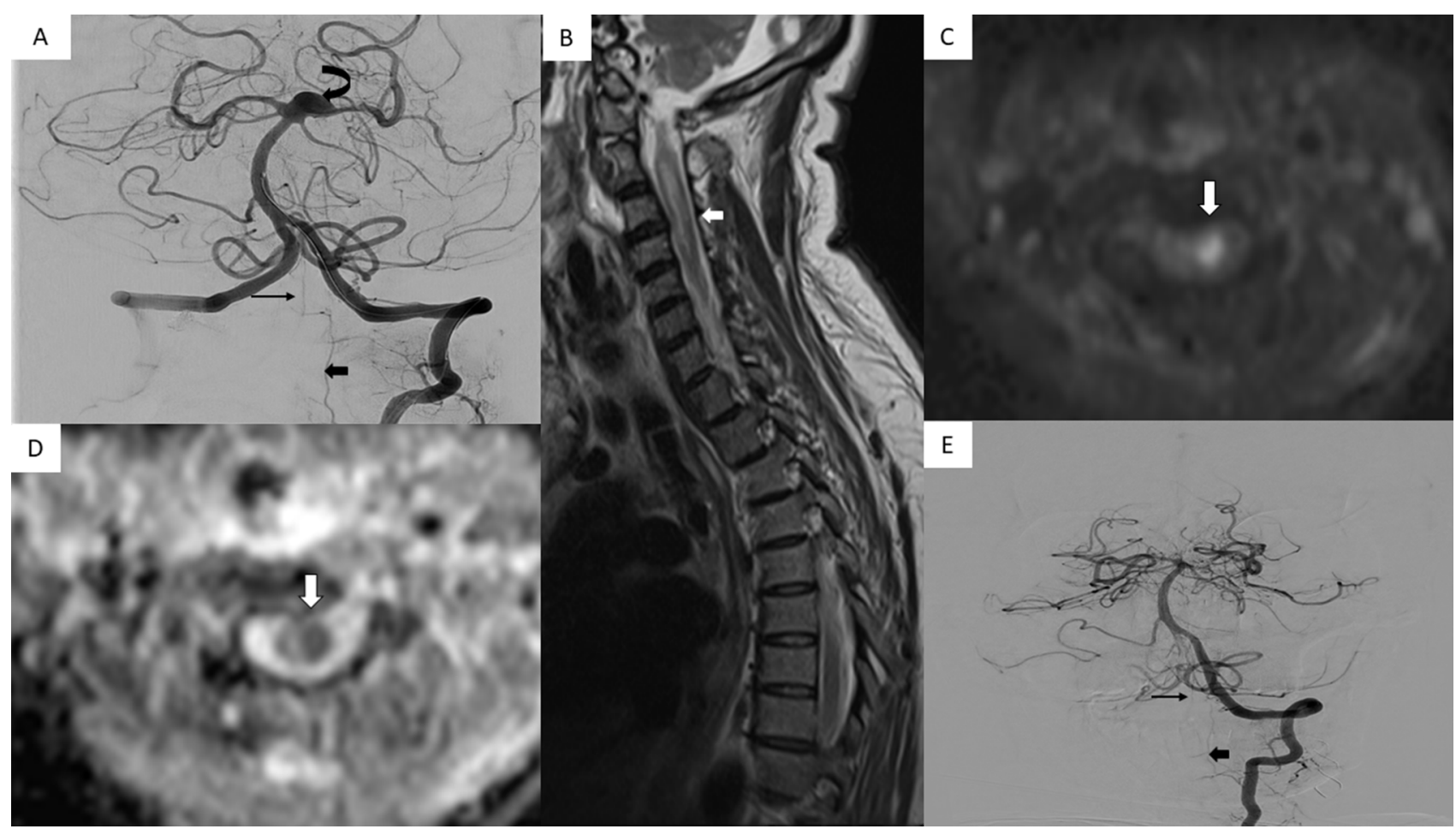

Fig. 2 (A) Left vertebral artery digital subtraction angiography frontal acquisition through guiding catheter reveals anterior spinal artery (ASA) (thin black arrow) with its radiculomedullary artery (RMA) reinforcement (thick black arrow) from the V2 segment of the vertebral artery. Basilar top aneurysm (curved arrow). (B) Spinal cord sagittal T2-weighted image demonstrating long segment (C2-C4) hyperintensity with a pencil-like configuration in its lower aspect (white arrow). (C and D) Trace diffusion-weighted imaging, apparent diffusion coefficient map depicting the left hemicord infarction involving both ASA and posterior spinal artery territory (white arrow). (E) Post-embolization acquisition reveals patent ASA (thin black arrow)with RMA reinforcement (thick black arrow).

but the patient was able to maintain saturation during treatment. The patient was referred for neurorehabilitation. At a 9-month follow-up, her modified Rankin Score was 3.

\section{Discussion}

$\mathrm{SCl}$ is an infrequent occurrence complicating aortic interventions /surgery, epidural injections, and spinal decompression. ${ }^{1}$ In the neuroendovascular realm, posterior circulation interventions have a theoretical risk of SCI. Between the coiling of VA aneurysms versus basilar aneurysms, a higher theoretical possibility of the SCI exists in the former due to inadvertent occlusion of the ASA. ${ }^{1,2}$ To best of our knowledge, only three such cases have been described in the literature. According to previous reports, wedging of the guiding catheter against the wall of VA with consequent flow stasis and thromboembolism into ASA may be a causal factor. ${ }^{3,4}$ The authors, however, described patent ASA on review of post-procedure DSA. We emphasize that the ASA and its RMA reinforcements were angiographically patent on review of both of our cases in the post-procedure angiograms, similar to previous reports. Stringent control of blood pressure was maintained with no significant intraprocedural fluctuation. We believe that pathogenetic mechanism may either be (a) small emboli beyond angiographic resolution that lodges into strategic territories or (b) local hemodynamic insufficiency of the RMA reinforcements of ASA due to local flow alteration caused by snuggly fitting guiding catheter in the VA. We reiterate that one of our patients developed $\mathrm{SCI}$ while on tirofiban. These observations corroborate with one of our hypotheses of hemodynamic insufficiency of the RMA reinforcements in the poorly collateralized ASA. Prior reports of SCI exist where intraoperative hypotension has been ascribed as a cause. ${ }^{5}$ Furthermore, the microvascular ischemia due to uncontrolled hypertension must have exacerbated the effect of hemodynamic insufficiency. Both our patients were in their 6th decade, similar to the previously reported cases., ${ }^{3,4}$ This suggests that advanced age may be a possible risk factor in the development of $\mathrm{SCI}$ in posterior circulation interventions apart from guiding catheter-induced local flow alteration and increased procedure time. The local anatomy and tortuosity of the origin of the VA significantly influence the distal navigation of the guide catheter and, therefore, the overall procedure time and success. ${ }^{6}$ No tortuosity at the ostia or the proximal VA was encountered in our cases.

A novel flow reversal method from VA to femoral vein via external shunt was devised and proposed by Ohshima et al to prevent post-procedural SCI. ${ }^{7}$ Nonetheless, its application is limited by the collateral status and its inability to reverse stagnated flow in the proximal VA.

Optimizing guiding catheter position, vasodilation, adequate heparinization, and minimizing procedure time hold potential to help avert such disastrous complications. 


\section{Conclusion}

$\mathrm{SCI}$ is a rare but potentially devastating complications due to guiding catheter-induced local flow alteration in posterior circulation interventions, especially in patients beyond the fifth decade. Categorically enough, it should be considered and excluded as a potential cause of post-procedural limb deficit associated with diaphragmatic palsy but without cranial nerve involvement in the case of posterior circulation neurointervention.

\section{Conflict of Interest}

None declared.

\section{References}

1 Zalewski NL, Rabinstein AA, Krecke KN, et al. Spinal cord infarction: clinical and imaging insights from the periprocedural setting. J Neurol Sci 2018;388:162-167
2 Tsuruta W, Yamamoto T, Ikeda G, et al. Spinal cord infarction in the region of the posterior spinal artery after embolization for vertebral artery dissection. Oper Neurosurg (Hagerstown) 2018;15(6):701-710

3 Iwahashi H, Fujita A, Tanaka H, Ikeda M, Morikawa M, Kohmura E. Spinal cord infarction after successful coil embolization of recurrent basilar bifurcation aneurysm: a case report. JNET 2018;12(8):398-403

4 Matsubara N, Miyachi S, Okamaoto T, et al. Spinal cord infarction is an unusual complication of intracranial neuroendovascular intervention. Interv Neuroradiol 2013;19(4):500-505

5 Yadav N, Pendharkar H, Kulkarni GB. Spinal cord infarction: clinical and radiological features. J Stroke Cerebrovasc Dis 2018;27(10):2810-2821

6 Gupta V, Basilar top aneurysm with extreme tortuosity: triaxial technique. In: Gupta V, Puri AS, Parthasarathy R, eds. 100 Interesting Case Studies in Neurointervention: Tips and Tricks. Springer Singapore; 2019:3-5

7 Ohshima T, Miyachi S, Matsuo N, et al. Novel vertebral artery flow reversal method for preventing ischemic complication during endovascular intervention. J Stroke Cerebrovasc Dis 2018;27(7):e144-e147 